\title{
A PRACTICAL APPROACH FOR MULTIMEDIA TRAFFIC MODELING
}

\author{
Timothy D. Neame, ${ }^{1}$ Moshe Zukerman ${ }^{1}$ and Ronald G. Addie ${ }^{2}$ \\ ${ }^{1}$ Department of Electrical and $\quad{ }^{2}$ Department of Mathematics \\ Electronic Engineering, \\ The University of Melbourne, \\ and Computer Science, \\ Parkville, Vic. 3052, Australia. \\ University of Southern Queensland, \\ Toowoomba, Qld. 4350, Australia. \\ \{t.neame, m.zukerman\}@ee.mu.oz.au addie@usq.edu.au
}

\begin{abstract}
This paper presents the M/Pareto process as a practical model for multimedia traffic. We explain the M/Pareto model, discuss its advantages and limitations, and demonstrate its ability to accurately predict the queueing performance of multimedia traffic.
\end{abstract}

Keywords: Multimedia traffic modeling, stochastic processes, queueing performance.

\section{INTRODUCTION}

Dimensioning and evaluation of traditional telephony networks is comparatively simple, with the well-known Erlang model accepted right around the world as the appropriate tool for the task. However traditional telephony is forming a smaller and smaller proportion of the total telecommunications load in most industrialised nations. Increasingly, packet-switched networks are replacing circuit-switched ones, and multimedia and data services are replacing simpler telephony services. While the Erlang model has served us well, it is simply not appropriate for this new environment.

We require a new model which is capable of consistently predicting the queueing behaviour of multimedia traffic. This model must be simple to use and give sufficiently accurate results. As yet, there is no widely accepted model which can be used in the modeling of multimedia and other modern traffic types. Many models have been proposed, but a consensus is needed. Meaningful comparison of results is impossible without such a consensus.

In this paper we propose the M/Pareto process as a suitable traffic model for this purpose. The M/Pareto process was presented in [8] as a model for long range dependent (LRD) traffic streams. By specifically taking LRD traffic into 
account, the M/Pareto process has a significant advantage over some of the other models which have been proposed, as there is now a large body of work which shows that multimedia and data traffic streams have this property of long range dependence $[6,7,12]$. Models which do not account for this property can still be used in certain specific situations, but are difficult to extend to general cases.

Recent work $[3,5,10]$ has shown that the M/Pareto process can accurately predict the queueing performance of measured Ethernet traffic and VBR video in a single server queue. The Internet is becoming an increasingly significant transport mechanism for multmedia content, so in this paper we consider the suitability of the M/Pareto process as a model for an IP packet stream.

In Section 2 we describe the modeling of packet-switched networks in general terms. We explain a framework based on modeling each link as a single server queue (SSQ), within which we examine suitability of the M/Pareto process. Section 3 describes the M/Pareto process itself. In Section 4 we explain the method we use to assign values to the parameters of the M/Pareto process so as to fit the queueing results of a given realistic traffic stream. In Section 5 we show that this method of fitting the M/Pareto process has given accurate estimates of queueing performance for Ethernet traffic streams and VBR video traffic. We extend these results to include a comparison with IP traffic in Section 6. In Section 7 we briefly discuss some obstacles which must be overcome to make the M/Pareto process a more useful traffic model.

\section{MODELING A LINK}

Regardless of the higher layer protocols being used, the single server queue (SSQ) forms a fundamental building block in the modeling of packet based networks. A packet-switched network can be considered to be a network of SSQs, with each link represented by an SSQ. We evaluate a given model in terms of its ability to characterise the buffer overflow probability of measured traffic in a SSQ for a range of threshold values and service rates. To do this, we measure the statistics of a given traffic stream, then fit the parameters of the model process so as to match the measured statistics. If the matched process consistently produces similar buffer overflow probability values as the modeled stream for a range of thresholds and service rates, then we say it is a good model of the given traffic stream.

If we consider each link to have a fixed maximum capacity, then the service rate of the SSQ representing that link will have a constant service rate. We use a $G / D / 1$ discrete time SSQ with an infinite buffer as our model for a link. We consider the queueing system to have a general arrival process $\left\{A_{n}\right\}$ which is assumed to be stationary and ergodic. Each value $A_{n}$ represents the amount of work arriving at the buffer in time interval $n$. In this paper we discuss two 
specific arrival processes - the Gaussian process and the M/Pareto process - and compare them with cases in which the $\left\{A_{n}\right\}$ sequence is given by measured traffic values.

We define the statistics of the input process as follows. Let the mean arrival rate be $\mu=\mathrm{E}\left(A_{n}\right)$, and let $A_{n}$ have variance $\sigma^{2}$. We assume that the process $A_{n}$ is long range dependent (LRD), with Hurst parameter $H$. The Hurst parameter represents the level of correlation in the process. We use the techniques described in [1] to estimate the Hurst parameter in the measured traffic streams.

The arrival process is fed into an SSQ with a constant service rate of $\tau$ per interval. For the infinite buffer queue the queueing curve is given by considering the values of the buffer overflow probability, $\operatorname{Pr}(Q>t)$, for a range of values for the threshold $t$.

\section{THE M/PARETO PROCESS}

It is now widely accepted that LRD streams are an important part of the traffic carried on broadband networks. Since LRD traffic, regardless of its source, is characterised by significant long bursts (see [12] and references therein), it is appealing to model LRD traffic using a model involving long bursts. The M/Pareto model is such a process, generating an arrival process based on overlapping bursts. The $\mathrm{M} /$ Pareto model described below is closely related to that given in [8], and is one of a family of such processes which form a sub-group of the $M / G / \infty$ models explored in [11].

$\mathrm{M} /$ Pareto traffic is composed of overlapping bursts. The duration of each burst is a random variable chosen from a Pareto distribution. Using the Pareto distribution here allows the significant long bursts which characterise LRD traffic to appear in the model traffic. The complementary distribution function for a Pareto-distributed random variable is given by

$$
\operatorname{Pr}(X>x)= \begin{cases}\left(\frac{x}{\delta}\right)^{-\gamma}, & x \geq \delta \\ 1, & \text { otherwise }\end{cases}
$$

$1<\gamma<2, \delta>0$. The mean of $X$ is $\frac{\delta \gamma}{(\gamma-1)}$ and the variance of $X$ is infinite. Note that the mean of the Pareto process in [5] was given incorrectly, and was a factor of $\gamma$ too small.

Burst arrivals are given by a Poisson process with rate $\lambda$. The arrival rate for each burst is constant for the duration of that burst, and has rate $r$. All bursts generate work at the same rate $r$. Thus the mean amount of work within one burst is: $\frac{r \delta \gamma}{\gamma-1}$. The mean total amount of work arriving from all bursts within an interval of length $t$ is

$$
\mu=\frac{\lambda t r \delta \gamma}{(\gamma-1)}
$$


Although the Pareto process has infinite variance, the variance of the M/Pareto process is finite. In [12] the term "Poisson burst process" was used to refer to processes like the M/Pareto process, where i.i.d. bursts of fixed rate start according to a Poisson process. For a Poisson burst process the variance function is given by repeatedly integrating the distribution function:

$$
\sigma^{2}(t)=2 \lambda r^{2} \int_{0}^{t} d t \int_{0}^{u} d u \int_{v}^{\infty} d x \operatorname{Pr}\{X>x\}
$$

Calculating for Pareto distributed burst durations gives

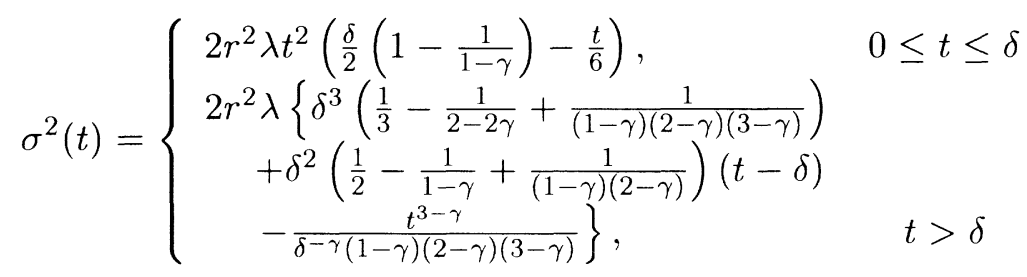

This corresponds with the variance function for processes of this type given in [2]. It represents a correction to the variance function quoted in $[3,5,10]$.

Examining the expression for the variance given in Equation (2), we see that for large $t$, the dominant term is $2 r^{2} \lambda \frac{\delta^{\gamma} t^{3-\gamma}}{(1-\gamma)(2-\gamma)(3-\gamma)}$. If we define $H=$ $\frac{3-\gamma}{2}$, then we can observe that for increasing $t$ the growth of this function is proportional to $t^{2 H}$. This implies that this model is asymptotically self similar with Hurst parameter

$$
H=\frac{3-\gamma}{2}
$$

The rate of the Poisson process, $\lambda$, controls the frequency with which new bursts commence. The superposition of two independent M/Pareto processes with identical burst length distributions will itself be an $\mathrm{M} / \mathrm{Pa}$-reto process with Poisson arrival rate equal to the sum of the arrival rates of the two constituent processes. Thus, increasing $\lambda$ can represent an increase in the number of sources making up an M/Pareto stream, and $\lambda$ can be thought of as representing the level of aggregation in the stream.

\section{MATCHING THE M/PARETO}

In the previous section we have seen that there are four parameters which define the behaviour of an M/Pareto process. These are: the Poisson arrival rate, $\lambda$; the arrival rate within a burst, $r$; the rate of decrease of the Pareto tail, $\gamma$; and the starting point of the Pareto tail, $\delta$. Equations (1), (2) and (3) define relationships between these four controlling parameters and three measurable statistics - the mean $(\mu)$, variance $\left(\sigma^{2}\right)$ and Hurst parameter $(H)$. 
Using these relationships, we can define M/Pareto processes which will produce given values of $\mu, \sigma^{2}$ and $H$. That means we can fit the M/Pareto process to match the statistics of an arbitrary real traffic stream. When fitting the M/Pareto in this way we have a system with three known values and four unknowns, so we can define an infinite number of $\mathrm{M} /$ Pareto processes with different parameters but which will all produce the same values of $\mu, \sigma^{2}$ and $H$. These processes will produce differing queueing performance.

In our analysis of the $\mathrm{M} / \mathrm{Pareto}$ process we consider families of $\mathrm{M} / \mathrm{Pa}$-reto processes with different Poisson arrival rates, but identical values of $\mu, \sigma^{2}$ and $H$. There are a number of ways we could achieve this. In this work we limit ourselves to trading off between the Poisson arrival rate for bursts, $\lambda$, and the cell arrival rate contributed by each burst, $r$. The parameters controlling the burst duration ( $\delta$ and $\gamma$ ) are held fixed. We do this so as to model increasing aggregation in the stream without altering the nature of the sessions making up the stream.

The Hurst parameter, $H$, depends only on the parameter $\gamma$, so altering $\lambda$ and $r$ will have no impact on that value. Given that we have already chosen $\delta$ and $\gamma$, the mean and variance values will be given by:

$$
\mu=C_{\mu} \lambda r \text { and } \sigma^{2}=C_{\sigma} \lambda r^{2}
$$

where $C_{\mu}$ and $C_{\sigma}$ are constants given by the values of $\delta, \gamma$ and the interval length, $t$. Clearly these two relationships imply that if we arrange the trade off between $\lambda$ and $r$ such that the mean is unchanged, the variance will be altered, and vice versa.

We take an approach in which we give first priority to fitting the variance. For each value of $\lambda$ we choose $r$ such that the variance is unchanged. Thus if $\lambda$ is multiplied by a factor $N$, then $r$ will be divided by a factor of $\sqrt{N}$. Making this adjustment will cause the mean to increase by a factor of $\sqrt{N}$. This prevents us from matching the statistics of a given measured process with a pure M/Pareto process with the same statistics. However, we can introduce a constant bit rate (CBR) component, $\kappa$, (which may be negative), so as to a match the mean arrival rate of the M/Pareto process to that of the modeled stream. The addition of $\kappa$ units of work per interval to every arrival interval will not affect the values of $\sigma^{2}$ or $H$, nor the queueing performance of the process.

\section{PREVIOUS RESULTS}

In previous work $[3,5,10]$ we have shown that the choice of $\lambda$ in the M/Pareto model has a significant impact on the queueing performance. Specifically, for identical values of $\mu, \sigma^{2}$ and $H$, different $\mathrm{M} /$ Pareto processes chosen according to the method described above yield different queueing curves. In [5] it was shown that as the value of $\lambda$ increases, the probability of buffer overflow 


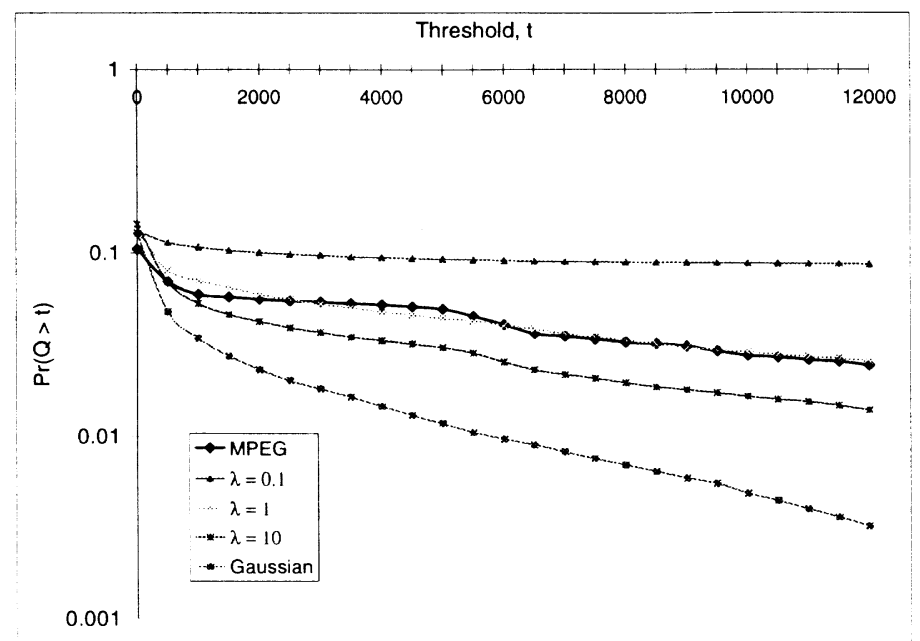

Figure 1 Fitting the M/Pareto process to a VBR video trace

converges to that given by a Gaussian process with the same mean, variance and Hurst parameter. Lower values of $\lambda$ give higher overflow probabilities.

This convergence to Gaussian is significant as it implies that as levels of aggregation increase, traffic may become more Gaussian in nature. If and when traffic becomes Gaussian, the analytic results given in $[2,4,5]$ will provide a simple basis for traffic modeling. Furthermore, the properties of the Gaussian process are such that when multiple Gaussian streams are aggregated together a multiplexing gain is possible.

However, studies of existing broadband networks, such as [9], have clearly shown that at present traffic is not Gaussian in nature. We suggest that, in data networks at least, this is because the level of aggregation in the stream is insufficient to reach Gaussian behaviour. We propose the M/Pareto process, with lower levels of aggregation (values of $\lambda$ ) as a model for this non-Gaussian traffic.

In [3] we showed that the M/Pareto model can accurately predict the queueing performance of an Ethernet trace when the right value for the parameter $\lambda$ is selected. We found that for lower $\lambda$ the M/Pareto process over-estimates the overflow probabilities of the Ethernet trace, but when $\lambda$ is chosen correctly the $\mathrm{M} /$ Pareto matches well.

In [10] we showed that matching the queueing curves of VBR video streams is also possible using the M/Pareto process. In this case, even though the modeled traffic is not an aggregated stream, the "level of aggregation" parameter, $\lambda$, must still be correctly chosen for an accurate modeling of the VBR video 


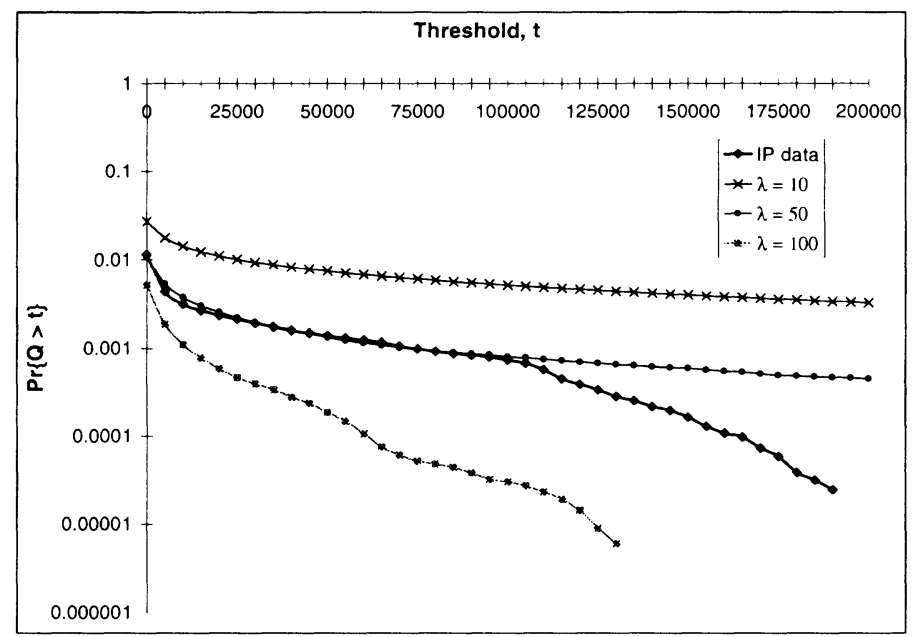

Figure 2 Matching the M/Pareto process to an IP trace

stream to be possible. Figure 1 shows the fitting of an M/Pareto process to one of the VBR MPEG sequences generated by Rose and analysed in [13].

\section{IP RESULTS}

In Figure 2 we show that the M/Pareto process sucessfully predicts the queueing performance of an IP traffic stream. The link traffic was recorded as a sequence of IP packet header summaries, which were reduced to a sequence of integers, where each value represents the number of bytes transmitted on the link in a 0.1 second interval. For this sequence, we measured a mean arrival rate of 5225 bytes per second and a variance of $21.233 \times 10^{6}$. We found $H \approx 0.90$. As for previous traffic streams, the correct value must be chosen for $\lambda$ before a fitting is possible. In this case $\lambda=50$ seems to give an accurate match.

The results given above show that the M/Pareto model can predict the queueing curves of both aggregated data traffic and VBR video streams. Accurate fitting is achieved only when the level of aggregation parameter, $\lambda$ is correctly assigned. An arbitrary matching the mean, variance and Hurst parameter of the $\mathrm{M} /$ Pareto process to that of the original traffic stream is not sufficient to accurately predict the queueing curve.

\section{LIMITATIONS OF THE M/PARETO}

We have seen that choosing the right value for $\lambda$ is vital in creating an $\mathrm{M} /$ Pareto process capable of matching the queueing curves of real traffic 


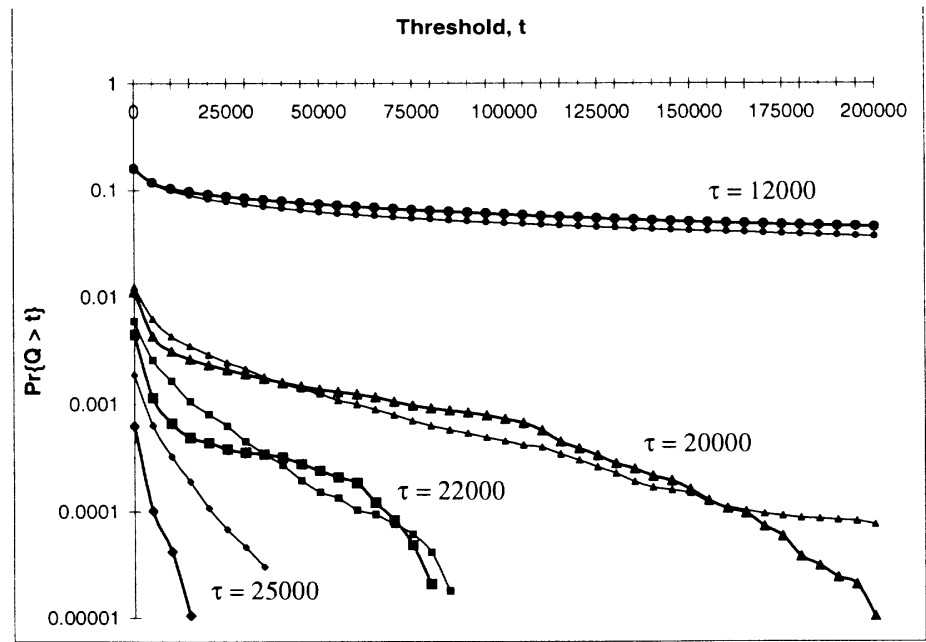

Figure 3 Comparing an M/Pareto with the IP trace for a range of service rates

streams. However the choice of $\lambda$ is complicated by the fact that the correct value of $\lambda$ differs depending on the service rate $\tau$.

Figure 3 shows that a single value of $\lambda$ may not be appropriate for all possible service rates. The figure shows four pairs of curves. In each pair the heavier line represents the queueing performance of the IP trace when fed into an SSQ with service rate $\tau$. The lighter line represents the performance of an M/Pareto process matched to the properties of the IP trace in an identical SSQ. The $\mathrm{M} /$ Pareto process used has $\lambda$ chosen so as to provide a good fit with the traffic when $\tau=20,000$, i.e. $\lambda=50$.

Choosing the correct value for $\lambda$ is not a trivial exercise. As yet we have no systematic method for determining the value of $\lambda$ to be used in modeling a given traffic stream. Trial and error must be used for each different modeled traffic stream, and for each different service rate. Until a heuristic for determining $\lambda$ is developed, the practical usefulness of the M/Pareto process will be limited.

The bursty nature of the M/Pareto process makes consistently estimating the queueing performance a difficult task. As yet we have no analytic expressions for the queueing performance of an M/Pareto process, so we must rely on simulation results. The LRD nature of the process means that long simulations are required to give meaningful accuracy in queueing results. However this will be true of any bursty process, and a bursty process is required to model bursty traffic, so any proposed model for bursty traffic will be similarly limited. 


\section{CONCLUSIONS}

In this paper we have shown that the $\mathrm{M} /$ Pareto process has much to recommend it as a model for multimedia traffic. We have seen that it can be used to accurately predict the queueing performance of traffic traces taken from both Ethernet and IP links. Between them, these protocols provide carriage for much of the multimedia content distributed today. We have also seen that the queueing performance of VBR video streams can be accurately predicted using the M/Pareto process.

We have seen that for the M/Pareto process to predict the queueing performance of these traffic types, the correct value must be chosen for the level of aggregation, $\lambda$, in the model. As yet, we do not have a simple formula or heuristic to determine the correct value for $\lambda$. If such a formula can be developed, the M/Pareto process will present a useful practical approach for the modeling of multimedia and data traffic.

\section{Acknowledgment}

The authors wish to thank Danielle Liu of AT\&T for supplying the IP packet trace analysed in this paper.

\section{References}

[1] P. Abry and D. Veitch. Wavelet Analysis of Long-Range-Dependent Traffic. IEEE Trans. on Information Theory, Vol. 44, No. 1, January 1998, pp 2-15.

[2] R. Addie, P. Mannersalo and I. Norros. Performance Formulae for Queues with Gaussian Input. Proceedings of ITC 16, June 1999, pp 1169-1178.

[3] R. G. Addie, T. D. Neame and M. Zukerman. Modeling Superposition of Many Sources Generating Self Similar Traffic. Proceedings of ICC '99, June 1999.

[4] R. G. Addie and M. Zukerman. An Approximation for Performance Evaluation of Stationary Single Server Queues. IEEE Trans. on Communications, December 1994.

[5] R. G. Addie, M. Zukerman and T. D. Neame. Broadband Traffic Modeling: Simple Solutions to Hard Problems. IEEE Communications Magazine, Vol. 36, No. 8, August 1998.

[6] J. Beran, R. Sherman, M. S. Taqqu, and W. Willinger. Long-RangeDependence in Variable-Bit-Rate Video Traffic IEEE Trans. on Communications, Vol. 43, No. 2/3/4, February/March/April 1995, pp 1566-1579. 
[7] W. E. Leland, M. S. Taqqu, W. Willinger, and D. V. Wilson. On the Self-Similar Nature of Ethernet Traffic (Extended Version). IEEE/ACM Trans. on Networking, Vol. 2, No. 1, 1994, pp 1-15.

[8] N. Likhanov, B. Tsybakov and N. D. Georganas. Analysis of an ATM Buffer with Self-Similar ("Fractal") Input Traffic. Proceedings of Infocom '95, April 1995.

[9] T. D. Neame, R. G. Addie, M. Zukerman, and F. Huebner. Investigation of Traffic Models for High Speed Data Networks. Proceedings of ATNAC '95, December 1995.

[10] T. D. Neame, M. Zukerman and R. G. Addie. Applying Multiplexing Characterization to VBR Video Traffic. Proceedings of ITC 16, June 1999.

[11] M. Parulekar and A. M. Makowski. Tail Probabilities for a Multiplexer with Self-Similar Traffic. Proceedings of Infocom '96, 1996.

[12] J. Roberts, U. Mocci, and J. Virtamo. Broadband Network Teletraffic, Final Report of Action COST 242. Springer, 1996.

[13] O. Rose. Statistical Properties of MPEG Video Traffic and Their Impact on Traffic Modeling in ATM Systems. Proceedings of the 20th Annual Conference on Local Computer Networks, October 1995. 